\title{
Fabrication de carbure de titane par combustion auto-entretenue de titane et de carbone : initiation par diffusion à l'état solide
}

\author{
D. VREL, J.-M. LIHRMANN and J.-P. PETITET
}

CNRS-LIMHP, Institut Galilée, avenue J.-B. Clément, 93430 Villetaneuse, France

\begin{abstract}
Self-propagating high temperature synthesis of titanium carbide from finely divided powders of $\mathrm{Ti}$ and $\mathrm{C}$ is initiated in the solid state by diffusion at the contacts between particles. In the model of concentric spheres of titanium and carbon, the formation of $\mathrm{TiC}$ has been computerized as a function of graphite particles radius $\mathrm{r}(\mathrm{m})$, time $\mathrm{t}(\mathrm{s})$ and absolute temperature $\mathrm{T}(\mathrm{K})$. The reaction rate $\alpha$ (moles of carbon reacted relative to the initial amount of carbon) can be expressed by the relationship :

\section{Introduction}

$$
\alpha=\left[\frac{6.453 \cdot 10^{-3}}{r^{2}} \cdot t \cdot \exp \left(-\frac{53683.46}{T}\right)\right]^{0.5325}
$$

L'élaboration de carbure de titane $(\mathrm{TiC})$ par réaction directe entre poudres finement divisées de titane et de carbone s'effectue par propagation auto-entretenue à travers les réactifs, après initiation à l'aide d'une source de chaleur (effet joule, pulse laser) [1,2]. Bien que la température de combustion à lintérieur du lit de poudres ait été mesurée à $2400^{\circ} \mathrm{C}$, soit plus de $700^{\circ} \mathrm{C}$ au-dessus du point de fusion du titane [3], la réaction est initiée par diffusion à l'état solide. On présente ici la contribution de la diffusion à l'état solide au mécanisme global, à partir d'un modèle idéalisé de sphères concentriques de Ti et $\mathrm{C}$.
\end{abstract}

\section{Modèle utilisé}

Entre $300 \mathrm{~K}$ et la température de fusion de $\mathrm{Ti}$ (1933 K, [4]), C et TiC ne subissent pas de variation allotropique, alors que la transition $\alpha \rightarrow \beta$ de Ti à $1155 \mathrm{~K}$ est négligée en raison de sa très faible influence sur l'évolution de la combustion [5]. Le diagramme de phases Ti-C est connu [6] ; TiC y est sous stoechiométrique en carbone et existe sous forme d'une solution solide étendue dans l'intervalle $\mathrm{TiC}_{0.5^{-}}$ $\mathrm{TiC}_{0.97}$

Dans le schéma diffusif considéré (fig. 1), TiC se forme à l'interface des sphères de $\mathrm{C}$ et de Ti. Le coefficient de diffusion de ${ }^{14} \mathrm{C}$ dans $\mathrm{TiC}$ est relativement insensible à la stoechiométrie, mais peut varier d'un facteur 10 suivant les auteurs. On adopte ici la valeur $D_{T i C}^{C}=4.54 \cdot 10^{-3} \cdot \exp \left(-\frac{-447300}{8.3144 T}\right)$, mesurée par [7]. D'après [8] le coefficient de diffusion de Ti dans TiC est constant pour C/Ti variant entre 0,67 et 0,97 , et vaut $4.35 \exp \left(-\frac{738800}{8.3144 T}\right)$. Il ressort que quelle que soit la température, le coefficient de diffusion de $\mathrm{Ti}$ dans $\mathrm{TiC}$ est inférieur à celui de $\mathrm{C}$ dans $\mathrm{TiC}$ d'un facteur voisin de $10^{5}$, et sera donc négligé. Le profil de concentration du carbone à travers l'interface $\mathrm{TiC}$ est fourni par le diagramme de phases, et la diffusion de $\mathrm{C}$ à travers $\mathrm{TiC}$ est traitée par la résolution des équations de Fick en coordonnées sphériques, à l'aide du schéma semi-implicite de Crank-Nicholson. 


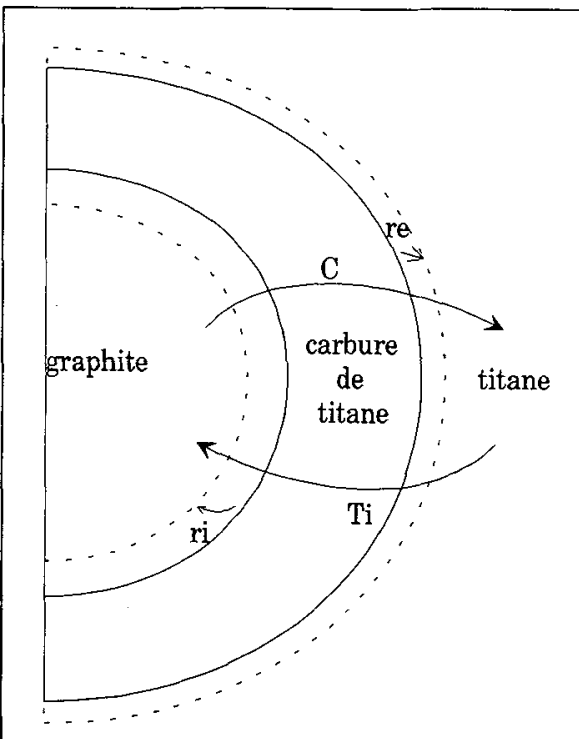

\section{Résultats}

\subsection{Initialisation}

Le degré d'avancement $\alpha$ de la réaction $\mathrm{Ti}+\mathrm{C} \rightarrow \mathrm{TiC}$ a été calculé à $1950 \mathrm{~K}$ pour une particule de graphite de rayon $1 \mu \mathrm{m}$, en adoptant comme condition initiale une couche interfaciale de $\mathrm{TiC}$ de volume successivement égal à $10^{-4}, 10^{-3}$ et $10^{-2}$ fois le volume du grain de graphite initial. Comme l'indique la figure 2 ci-dessous, les trois courbes rejoignent une droite commune dès que le degré d'avancement dépasse la condition initiale, c.a.d. $\alpha>10^{-4}$ pour la courbe supérieure, $\alpha>10^{-3}$ pour la courbe intermédiaire et $\alpha>10^{-2}$ pour la courbe inférieure. Les calculs ultérieurs ont été effectués avec la condition initiale $10^{-3}$.

Fig. 1 : Schéma du modèle de diffusion

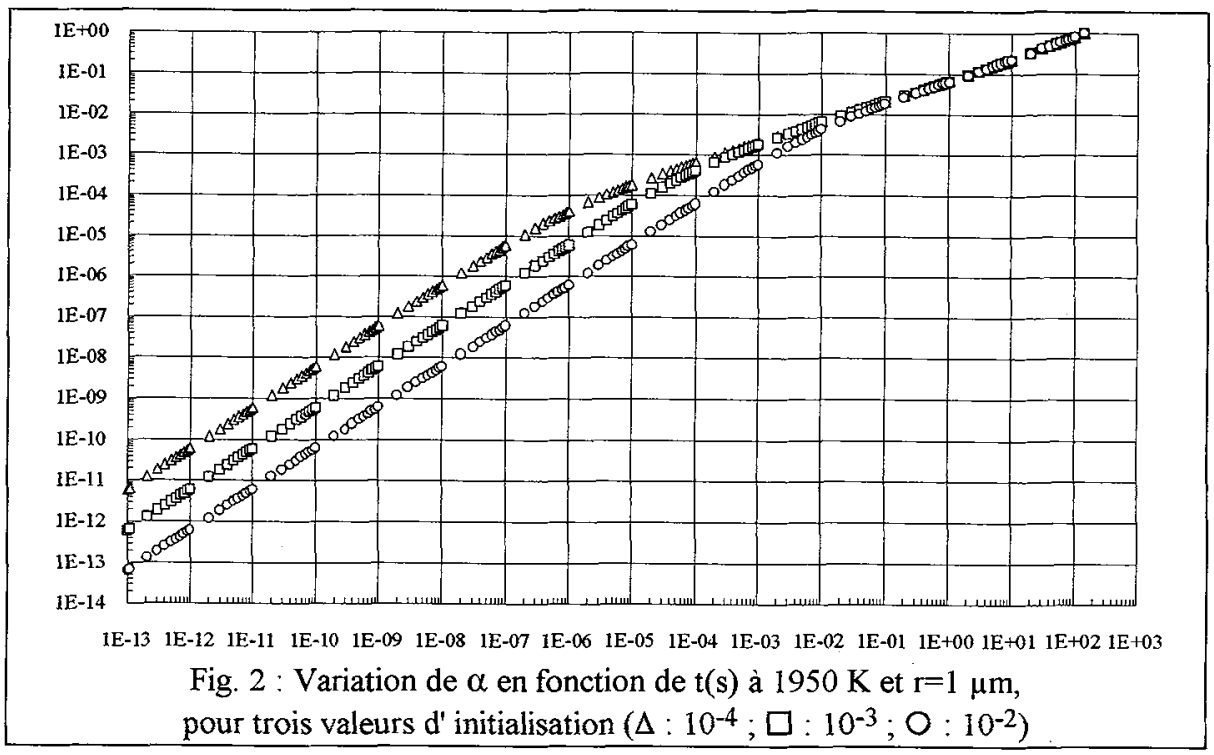

\subsection{Influence du temps}

Le degré d'avancement de la réaction a ensuite été calculé en fonction du temps, pour $\mathrm{r}=1 \mu \mathrm{m}$ et pour des températures variant entre $1200 \mathrm{~K}$ et $1950 \mathrm{~K}$ par pas de $25 \mathrm{~K}$. La figure 3 indique que toutes les courbes tendent vers des droites parallèles de pente $\xi=0.5325$, ce qui permet d'écrire $\alpha(t, T)=F(T) \cdot t^{\xi}$, avec $F(T)=\left[\frac{1}{t_{\infty}(T)}\right]^{\xi}\left(t_{\infty}(T)\right.$ est le temps au bout duquel $\alpha=1$ à la température $\left.T\right)$. 


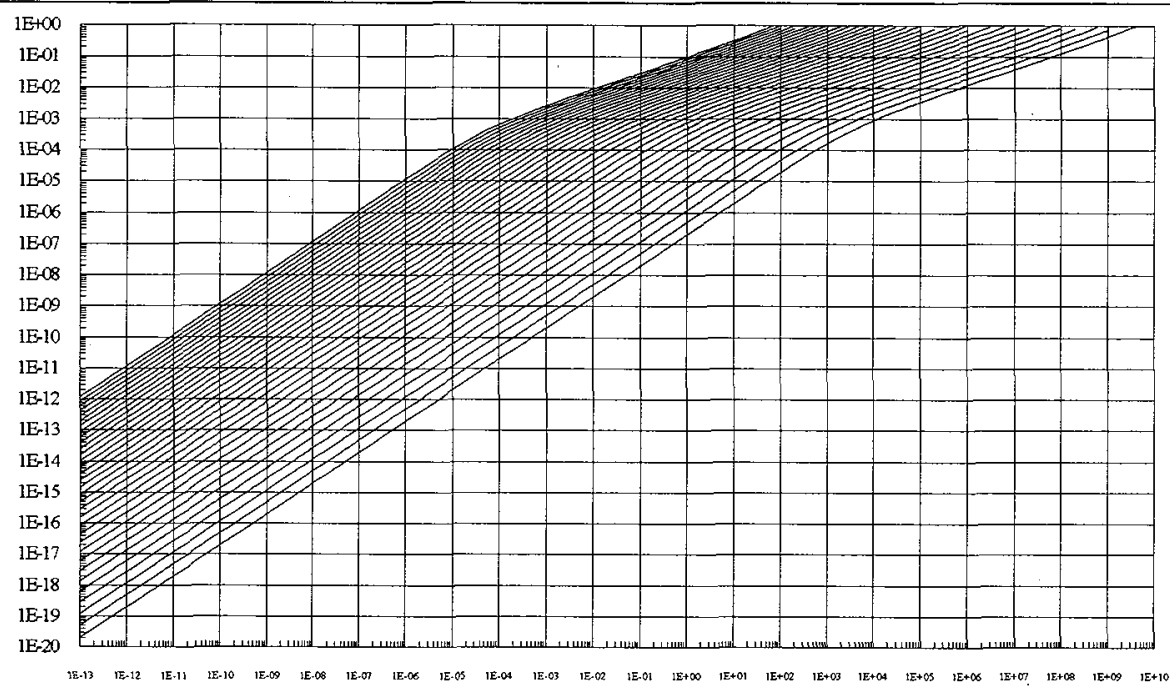

Fig. 3 : variation de $\alpha$ en fonction de $t(s)$ pour $r=1 \mu \mathrm{m}$ et $\mathrm{T}$ variant de $1200 \mathrm{~K}$ (en bas) à $1950 \mathrm{~K}$.

\subsection{Influence de la température}

Celle-ci est fournie par l'étude de $t_{\infty}$ en fonction de la température. La figure 4 indique, pour $r=1 \mu \mathrm{m}$, une variation linéaire du logarithme népérien de $\frac{1}{\mathrm{t}_{\infty}}$ en fonction de $\frac{10^{3}}{T}$, d'où la relation $\frac{1}{t_{\infty}(T)}=C \cdot \exp \left(-\frac{A}{T}\right)$. La pente $A$ de la droite est mesurée à 53683.46 , ce qui correspond, à $0,2 \%$ près, à la valeur $\frac{E^{*}}{R}=\frac{447300}{8.3144}$.

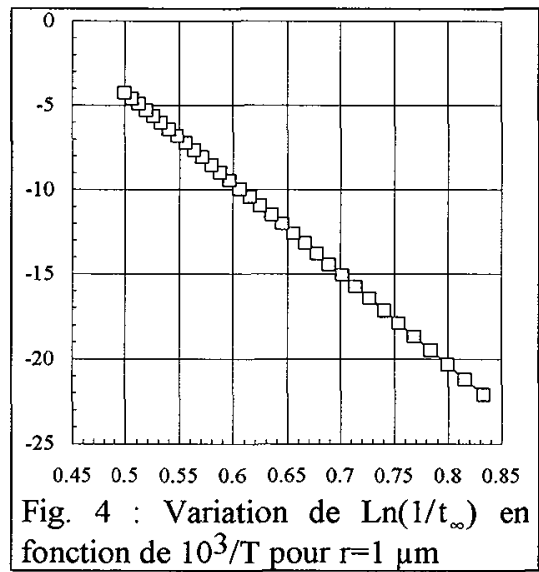

\subsection{Influence du rayon $r$ des particules de graphite.}

Une modélisation identique à celle exposée en 3.2. a été effectuée pour une valeur de $r=2 \mu \mathrm{m}$. On calcule à partir de la figure 5 une pente identique à celle fournie par la figure 3 , donc l'exposant $\xi$ peut être considéré comme indépendant de $\mathrm{r}$. C'est également le cas de A que l'on assimile à $\frac{E^{*}}{\mathrm{R}}$. Il s'ensuit que l'influence de $r$ dans l'expression $\alpha=\left[\mathrm{C} \cdot \mathrm{t} \cdot \exp \left(-\frac{\mathrm{A}}{\mathrm{T}}\right)\right]^{\xi}$ est contenue essentiellement dans le paramètre $\mathrm{C}=\frac{1}{\mathrm{t}_{\infty}(\mathrm{T})} \cdot \exp \left(+\frac{\mathrm{A}}{\mathrm{T}}\right)$, indépendant de la température.

On a alors calculé $\mathrm{C}$ pour des valeurs de $\mathrm{r}$ variant de $1 \mu \mathrm{m}$ à $100 \mu \mathrm{m}$ par pas de $1 \mu \mathrm{m}$. Comme l'indique la figure $6, \mathrm{C}$ varie linéairement en fonction de $\ln (\mathrm{r})$, avec une pente égale à -2 et une ordonnée pour $\mathrm{r}=1 \mu \mathrm{m}$ égale à $6.45010^{9} \mathrm{~s}^{-1}$, d'où l'expression de $\mathrm{C}\left(\mathrm{s}^{-1}\right)$ :

$$
C=\frac{6.450 \cdot 10^{-3}}{[\mathrm{r}(\mathrm{m})]^{2}}
$$




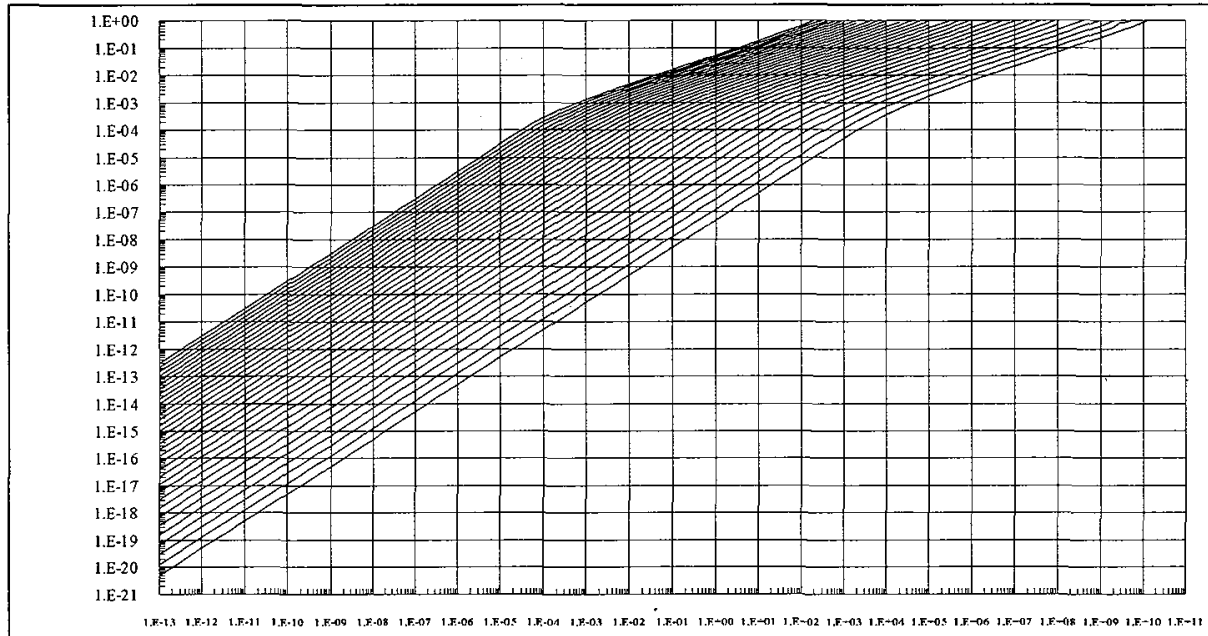

Fig. 5 : Variation de $\alpha$ en fonction de $\mathrm{t}(\mathrm{s})$ pour $\mathrm{r}=2 \mu \mathrm{m}$ et $\mathrm{T}$ variant de $1200 \mathrm{~K}$ (en bas) à $1950 \mathrm{~K}$

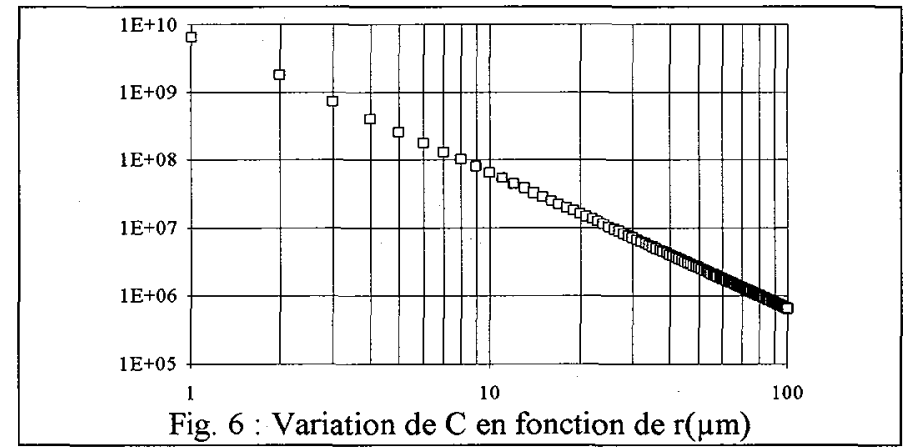

On en déduit l'expression finale de $\alpha$, degré d'avancement de la réaction $\mathrm{Ti}+\mathrm{C} \rightarrow \mathrm{TiC}:$

$$
\alpha(\mathrm{r}, \mathrm{t}, \mathrm{T})=\left[\frac{6.450 \cdot 10^{-3}}{[\mathrm{r}(\mathrm{m})]^{2}} \cdot \mathrm{t}(\mathrm{s}) \cdot \exp \left(-\frac{53683.46}{\mathrm{~T}(\mathrm{~K})}\right)\right]^{0.5325}
$$

A $1950 \mathrm{~K}$ et $\mathrm{r}=1 \mu \mathrm{m}$, le calcul donne, pour un temps de $1 \mathrm{~s}$, une valeur $\alpha=7.2010^{-2}$, ce qui confirme la contribution très partielle de la diffusion en phase solide à la formation de TiC (l'essentiel de la réaction a lieu en phase liquide, jusqu'à $2670 \mathrm{~K}$ ).

\section{Références}

[1] Lihrmann J.-M., Hervé P. et Petitet J.-P., Proceedings 2nd Conf. Europ. Ceram. Soc.,

Augsburg, ed. DKG, à paraître

[2] Vrel D.,Lihrmann J.-M. et Petitet J.-P., L'industrie Céramique, 875 (1992) 680-3

[3] Hervé P., Tobaly P., Lihrmann J.-M. et Petitet J.-P., In : Calorimétrie et analyse thermique, vol XXIII, ed. D. Clausse, UTC, 1992, 113-118

[4] JANAF Thermochemical Tables, 2nd ed., 1971.

[5] Merzhanov A.G., Arch. Combust., 1 (1981) 23

[6] Storms E.K., The Refractory Carbides, Academic Press, New York, 1967, p.3

[7] Sarian S., J. Appl. Phys., 39 (1968) 3305

[8] Sarian S., J. Appl. Phys., 40 (1969) 3515 